\title{
Problems And Solutions For Collector Systems Of Powerful Gyrotrons
}

\author{
V.E.Zapevalov \\ Institute of Applied Physics, Nizhny Novgorod, Russia, zapev@appl-sci-nnov.ru
}

Serious problem of high-power gyrotrons is high level of the energy of the spent electron beam after generation of microwave radiation. In a typical high-power continuous mode gyrotron with an electron beam power of $3 \mathrm{MW}$ after generation of $1 \mathrm{MW}$ microwave radiation (which corresponds to $33 \%$ efficiency), the power of $2 \mathrm{MW}$ will be concentrated in the spent electron beam, which should be scattered by collector [1-4]. Without the use of special actions to o increase the current landing collection area, the power density at the collector is excessive and leads to its rapid damage. The use of CPD collectors somewhat improves the situation [1-4]. Typical efficiency gyrotron without CPD is $30-35 \%$, and with CPD about $50 \%$. In addition to a very noticeable increase in efficiency, the use of a CPD collector greatly reduces the power dissipated on the collector. Thus, with an increase in efficiency from $33 \%$ to $50 \%$, in a megawatt gyrotron, the power at the collector is halved. The collector is the most massive, large-sized and very expensive subsystem of powerful gyrotrons. Nevertheless, the collector is still one of the most frequently damaged parts of the gyrotron. In addition to the collector itself, the collection system includes a magnetic system for transporting the electron flow, a HV power supply system, a cooling system that provide intense cooling water flows (about $1 \mathrm{~m}^{3} / \mathrm{s}$ per 1 MW of power), an overload protection system, ets.

A review of variants of collector systems from the point of view of electron optics is given in [5]. Later we will dwell on the simplest systems that are close to practical implementation or are already in use at the present time. In most powerful gyrotrons with continuous or long-pulse operation collectors with longitudinal scanning of the electron beam are used [6]. Such a gyrotron as an electronic device is shown in FIG. 2, contains an electron gun and an electro-dynamic system (not shown in the diagram), a collector - 1 with a current-receiving surface -2 in the form of a symmetrical body, a vacuum case - 8 (corpus), a magnetic system - 9 for transporting of an electron beam, and an additional solenoid 3 , fed by a periodically varying current. The electron beam 4 in the region of the collector 1 with the minimum field of the additional solenoid moves along the trajectory 5 , with the negative field of the additional solenoid along trajectory 6 , and for the maximum value of the field along trajectory 7 . Thus, the electron beam 4 scans due to the action of the alternating magnetic field of the additional solenoid 3 on the current-receiving surface. As a result, the scanning zone of the electron beam 4 can significantly exceed the zone of the beam settling in the absence of the alternating field of the additional solenoid 3. Reduction of the heat flux density on the current receiving surface of the collector and, thus, the temperature of this surface is achieved by distributing the electron beam over a larger surface area of the collector with a time-varying magnetic field produced by an additional solenoid 3 surrounding the collector; a current receiving surface is profiled. The analog of this technical solution is the electron microwave device [7], where the uniformity of the heat flow density along the surface of the collector is achieved in this case by profiling the thickness of the winding of an additional solenoid, or fast transversal field [9].

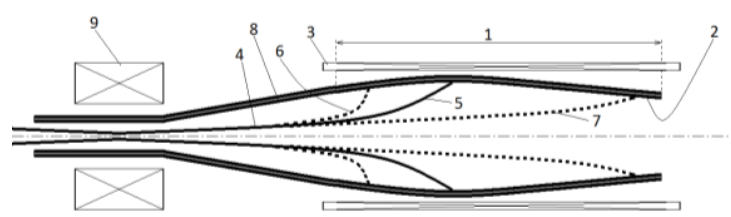

Fig. 1. A typical scheme of a powerful gyrotron with a collector, using longitudinal scanning.

The qualitative dependence of the temperature of an arbitrary point of the current-sensing collector surface in the steady-state continuous mode on time is shown in Fig.2. It can be seen that this method has a significant disadvantage: the maximum temperature of the current receiving surface is significantly higher than that could be obtained with a static distribution of the heat flux over the entire settling zone, the scanning principle itself implies a periodic effect of an electron beam on an arbitrary point of current-sensing surface of the collector and, consequently, the presence of temperature fluctuations of this point in time with the scanning frequency. The range of these oscillations obviously decreases with increasing scanning frequency (see Fig. 2).

But the scanning frequency is limited because of the screening of the alternating magnetic field by the conducting collector. Vacuum casing is made of a material with high electrical conductivity; the collector of the device is simultaneously a part of the vacuum casing. In practice, the scanning frequency is not more than a few Hertz, which is clearly not enough to eliminate temperature fluctuations.

For the body of such a collector, close in shape to a long thin nonmagnetic cylindrical shell, the characteristic frequency at the beginning of a significant screening is [8]:

$$
f_{\text {shield }}=\frac{1}{\pi \sigma \mu_{0} R h},
$$

where, $\mu_{0}=4 \pi \cdot 10^{-7} \Gamma H / \mathcal{M}, \sigma$ - is the conductivity of the material of the collector 1 , in $\mathrm{Ohm}^{-1} \mathrm{~m}, R$ is the radius of the collector $1, m, h$ is the thickness of the collector $1, m$. 


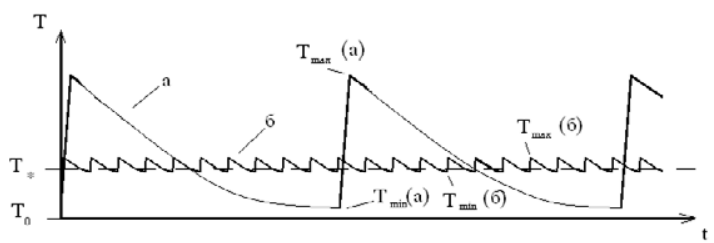

Fig. 2. The change in the temperature of the section of the collector surface versus time at a slow and fast scanning frequency

In [10], a collector is proposed in the form of an isolated single or multi-spiral coil made of a pipe through which a cooling liquid flows. For a helix in which closed circular currents are impossible, the characteristic circular frequency of the beginning of a significant screening coincides with the skin-effect frequency

$$
f_{\text {skin }}=\frac{1}{\pi \sigma \mu_{0} h^{2}}
$$

Thus, this solution allows, for example, to increase the scanning frequency by a factor of $\frac{f_{\text {skin }}}{f_{\text {shield }}}=\frac{R}{h}$ compared to the above described collector (all other things being equal). For a typical gyrotron copper collector $\left(R_{\text {coll }}=10 \mathrm{~cm}\right.$ and thickness $h=5$ $\mathrm{mm}$ ), the frequency gain will be 20 times. Thus, the use of a spiral gives a noticeable gain in the allowable scanning frequency. This will significantly reduce the maximum temperature of the surface of the collector at the same power of the spent beam, and significantly increase the longevity of the instrument or significantly increase the permissible value of the power of the spent electron beam with the same longevity of the device.

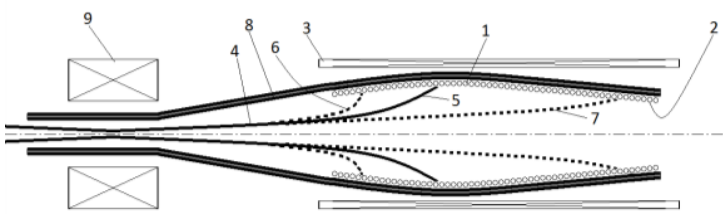

Fig. 3. Scheme of a powerful gyrotron with a spiral collector, using longitudinal scanning.

Another option is the use of a collector in which an external surface of the symmetrical body is used for current-landing (see Fig. 4) [11]. In this case, the housing of the electronic device can be made of a material with a low electrical conductivity (for example stainless steel) and the shielding is significantly weakened.

$$
f_{\text {corp }}=\frac{1}{\pi \sigma_{\text {corp }} \mu_{0} R_{\text {corp }} h_{\text {corp }}},
$$

where $\sigma_{\text {corp }}$ is the conductivity of the material of the vacuum body 1 of the microwave device, $R_{\text {corp }}$ - its radius in $m$, and $h_{\text {corp }}$ - its thickness in $m$. Thus, it is possible to increase the scanning frequency by a fac- tor of $\frac{f_{\text {corp }}}{f_{\text {coll }}}=\frac{\sigma_{\text {coll }} R_{\text {coll }} h_{\text {coll }}}{\sigma_{\text {corp }} R_{\text {corp }} h_{\text {corp }}}$ compared to the variant

Fig. 1 (all other conditions being equal).

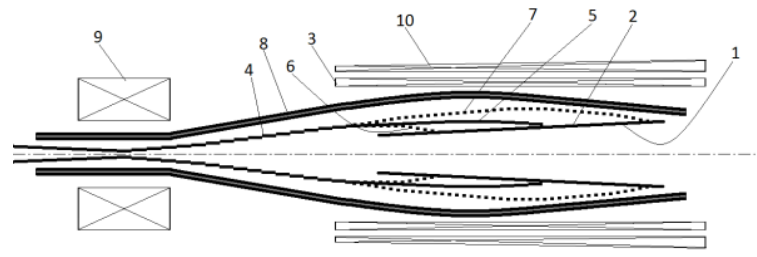

Fig. 4. The scheme of a powerful gyrotron with a collector inside the electron beam, using longitudinal scanning.

For example, for a typical powerful gyrotron, a collector made of copper $\left(\sigma_{\text {coll }}=60 \cdot 10^{6} \mathrm{ohm}^{-1} \mathrm{~m}\right)$ has a radius of $100 \mathrm{~mm}$ and a thickness of $5 \mathrm{~mm}$. Let the device have the same internal collector. For example, for $R_{\text {coll }}=110 \mathrm{~mm}$ (material stainless steel 12X18H10T $\left(\sigma_{\text {corp }}=1,4 \cdot 10^{6} \mathrm{ohm}^{-1} \mathrm{~m}\right)$ ), and $h_{\text {corp }}=2 \mathrm{~mm}$. The gain in the maximum allowable scanning frequency will be almost two orders of magnitude.

\section{References}

1. A.V. Gaponov, et al., Author's certificate No. 223931 "A device for generating electromagnetic oscillations of the centimeter, millimeter, and submillimeter wavelength ranges."

2. G.G.Denisov, et al. Development in Russia of highpower gyrotrons for fusion. // Nuclear Fusion, 48, №5, 2008, 5 pp.1-5.

3 M. Thumm, State-of-the-Art of High Power GyroDevices and Free Electron Masers, Update 2015 (KIT Scientific Reports; 7717), 2016. https://doi.org/10. $\underline{5445 / \mathrm{KSP} / 1000055471}$

4 A.G. Litvak et al., Development in Russia of megawatt power gyrotrons for fusion, // J. Infrared Millim. Terahertz Waves 32 (3) (2011) 337-342, https://doi.org/10. 1007/s10762-010-9743-8.

5. V.N. Manuilov, et al, Gyrotron collector systems: Types and capabilities // Infrared Physics and Technology, 91 (2018) 46-54. doi:10.1016/j.infrared.2018.03.024

6. Yu D. Larichev, A. Sh Fiks, The Microwave Device, Author's Certificate of USSR, Patent SU 1238617 A, USA.

7. F.Faillon, G.Mourier et al. Elecron collector for electron tubes. U S Patent Number 4,933,594. Date June 12, 1990.

8.V.V. Vasiliev, et al. Conductive shells in a pulsed electromagnetic field. M., // Energoatomizdat, 1982, p.879 .

9. V.Erckmann, G.Dammertz, M.Schmid. Patent EP2150965A1 - Method and apparatus for collector sweeping control of an electron beam 2010-02-10

10. Zapevalov V.E., Fix A.Sh. IAP RAS, Electron microwave device, Patent No 2576391, Application: 2014146356/07, 18.11.2014, (priority 18 November 2014), Published: 10.03.2016 Bul. № 7

11. Zapevalov V.E., Fix A.Sh, IAP RAS, Electronic microwave device, Patent No. 2630251 Application No. 2016113110 , priority date $05 / 04 / 2016$. The date of state registration in the State Register on September 6, 2017. 